\title{
Experiencia en estapedostomías en Hospital del Salvador entre 2012 y 2018
}

\section{Stapedotomy experience at Hospital del Salvador between 2012 and 2018}

\author{
Leandro Rodríguez $\mathbf{H}^{1,2,4}$, Paula Silva $\mathbf{R}^{2}$, José Tomás Zemelman $\mathrm{L}^{2}$, Rocío Carrasco $\mathrm{C}^{3}$, \\ Mauricio Silva $C^{1,2}$, Tamara Barría $\mathrm{E}^{1}$, Cristián Carriel $\mathrm{P}^{1}$, Alejandro 0jeda $\mathbf{S}^{1,2}$.
}

\begin{abstract}
RESUMEN
Introducción: La otoesclerosis es una enfermedad de presumible origen metabólico que determina la fijación de la cadena osicular. La estapedostomía con instalación de prótesis es la cirugía de elección en la actualidad para esta patología.

objetivo: Describir experiencia y resultados del tratamiento quirúrgico de pacientes con otoesclerosis en el Hospital del Salvador entre los años 2012 y 2018.

Material y método: Estudio descriptivo retrospectivo mediante revisión de fichas clínicas de pacientes intervenidos quirúrgicamente en nuestro servicio desde enero de 2012 hasta septiembre de 2018 con sospecha de otoesclerosis.

Resultados: Se realizaron en total 52 cirugías a 45 pacientes, 40 vía microscópica, 10 vía endoscópica y 2 mixtas, se utilizaron dos modelos de prótesis, Schuknecht - type piston and wire y Stapes-Prothesen. El promedio tonal puro (PTP) por vía aérea prequirúrgico fue de $65 \mathrm{~dB}$, con una diferencia ósea-aérea (gap) promedio de $36 \mathrm{~dB}$. El PTP promedio posquirúrgico fue de $35 \mathrm{~dB}$ con gap promedio de $9 \mathrm{~dB}$, logrando en promedio una mejoría de $31 d B$ en la vía aérea y $27 d B$ de cierre de gap. El 92\% de los pacientes intervenidos lograron mejoría con respecto al gap posoperatorio.

Conclusión: La estapedostomía es una intervención que si bien no está exenta de complicaciones, ha demostrado ser segura y tener buenos resultados auditivos en la mayoría de los pacientes.
\end{abstract}

Palabras clave: Otoesclerosis, estapedostomía.

\begin{abstract}
Introduction: Otosclerosis is a presumable metabolic disease that determines ossicular chain fixation. Stapedotomy with prosthesis installation is the surgery of choice for this pathology at present.
\end{abstract}

\footnotetext{
1 Servicio de Otorrinolaringología, Hospital del Salvador, Santiago, Chile.

2 Departamento de Otorrinolaringología, Hospital del Salvador, Facultad de Medicina, Universidad de Chile, Santiago, Chile.

3 Escuela de Medicina, Facultad de Medicina, Universidad de Chile, Santiago, Chile.

4 Servicio de Otorrinolaringología, Clínica Universidad de Los Andes, Santiago, Chile.

Los autores declaran no tener conflictos de interés.
}

Recibido el 4 de diciembre, 2019. Aceptado el 10 de febrero, 2020. 
Aim: To describe the experience and results of the surgical treatment of patients with otosclerosis in the Hospital del Salvador between 2012 and 2018.

Material and methods: Clinical records were reviewed of patients treated surgically in our department from January 2012 to September 2018 with suspicion of otosclerosis.

Results: A total of 52 surgeries were performed in 45 patients, 42 via microscopy and 10 via endoscopy, two models of prostheses were used Schuknecht - type piston and wire y Stapes-Prothesen. The pure tonal average (PTP) by pre-surgical air was 65 $d B$, with an average air gap (gap) of $36 d B$. The average post-surgical PTP was $35 d B$ with an average gap of $9 \mathrm{db}$, achieving on average an improvement of $31 \mathrm{~dB}$ in the airway and $27 \mathrm{~dB}$ of gap closure. $92 \%$ of the patients who underwent surgery improved with respect to the postoperative gap.

Conclusion: The stapedotomy is an intervention that although not free of complications, has been shown to be safe and have good hearing results in most patients.

Key words: Otosclerosis, stapedotomy.

\section{INTRODUCCIÓN}

La otoesclerosis es una enfermedad metabólica de etiología desconocida que afecta aproximadamente al $1 \%$ de la población'. Posee una transmisión autosómica dominante con baja penetrancia y expresividad variable, aunque también puede ser de aparición esporádica. Se caracteriza por generar fijación de la cadena osicular y con ello hipoacusia de conducción, pero se ha descrito que hasta un tercio de los pacientes desarrollan finalmente hipoacusia mixta². La edad de presentación es entre la segunda y tercera década de la vida, existiendo un predominio en mujeres con una relación de 2:1 respecto a los hombres ${ }^{1}$. La clínica se caracteriza por una hipoacusia de conducción lentamente progresiva, bilateral en el $80 \%$ de los casos y asimétrica. Algunos pacientes también refieren tinnitus. A la otoscopía el tímpano se encuentra normal en la mayoría de los casos, pero también puede presentar una coloración roja sobre el promontorio 0 en la zona anterior a la ventana oval, conocida como el signo de Schwartze, que solo se ve en el inicio de la enfermedad y corresponde a las zonas de neovascularizacion ${ }^{2,3}$.

En la audiometría se observa una hipoacusia de conducción, o mixta si hay compromiso coclear. También puede encontrarse el signo "notch de Carhart" que consiste en una baja en la vía ósea en las frecuencias de los $2 \mathrm{kHz}$ acercándose a la vía aérea ${ }^{3}$. En la impedanciometría se puede encontrar una curva $A$ o As, efecto on-off y reflejos invertidos, lo que se puede ver en un inicio de la enferme- dad, para posteriormente transformarse en reflejos negativos ${ }^{4}$. Con respecto al estudio imagenológico, en la tomografía computarizada de oídos, se observan zonas de menor densidad en relación a la ventana oval, la platina o el resto de la cóclea si se trata de una otoesclerosis coclear, siendo un examen fundamental para el diagnóstico diferencial y la planificación de la cirugía' ${ }^{1}$. La estapedostomía con instalación de prótesis es la cirugía de elección en la actualidad para esta patología, demostrando una alta eficacia, bajo índice de complicaciones y un resultado estable a largo plazo $0^{5}$.

\section{OBJETIVO}

Describir la experiencia y resultados del tratamiento quirúrgico de pacientes con otoesclerosis en el Hospital del Salvador entre los años 2012 y 2018.

\section{MATERIAL Y MÉTODO}

Se realizó un estudio descriptivo retrospectivo mediante revisión de fichas clínicas de pacientes intervenidos quirúrgicamente en nuestro servicio desde enero de 2012 hasta septiembre de 2018 con sospecha de otoesclerosis. Este trabajo de investigación fue aprobado por el Comité de Ética Científico Adultos del Servicio de Salud Metropolitano Oriente. Se obtuvo el registro de 52 casos operados de estapedostomía entre los años 2012 y 2018 en el Hospital del Salvador. De éstos, 
se disponía de 49 casos con registro completo que cumplieran los siguientes criterios de inclusión:

Historia clínica compatible con otoesclerosis

Otoscopía normal

Audiometría con hipoacusia de conducción con gap $\geq 30 \mathrm{~dB}$

Impedanciometría con curvas A o As

Tomografía computarizada de oídos con protocolo de otoesclerosis (cortes de 0,6 mm)

Sin antecedente de otitis media crónica

Se excluyó a pacientes con gap menor 300 aquellos en que los hallazgos imagenológicos o intraquirúrgicos no fueran compatibles con otoesclerosis.

En las cirugías realizadas se utilizó el siguiente equipamiento quirúrgico: Microscopio Carl Zeiss s8 $\mathrm{f} 170$, endoscopio rígido Storz ${ }^{\circledR}$ de $4 \mathrm{~mm}$ y 2,7 $\mathrm{mm}$ de $0^{\circ}$, de $14 \mathrm{~cm}$, una torre de video Storz ${ }^{\circledR}$ con cámara full HD, un Skeeter dril ${ }^{\otimes}$ de Medtronic con fresa de 0,8 mm, una consola Integrated Power Console de Medtronic ${ }^{\circledR}$ (IPC system), prótesis tipo Schuknecht de fluoroplastic de $0,6 \mathrm{~mm}$ de diámetro y de 4,25 - 4,5 - 4,75 mm de largo según la medición.

\section{Técnica quirúrgica}

Bajo anestesia total intravenosa se posiciona al paciente en decúbito dorsal con hombro ipsilateral al oído a intervenir bajo la posición de su cabeza. El cirujano se encuentra sentado con ambos codos apoyados en la mesa quirúrgica con la torre endoscópica al frente. Se colocan cotonoides en el conducto auditivo externo con vasoconstrictor y se infiltra con lidocaína/epinefrina al 1:100.000 en el conducto auditivo externo por vía endoaural. Posteriormente, bajo visión microscópica 0 endoscópica de $0^{\circ}$ se realiza incisión vía endoaural en forma de rectángulo de las 12 a las 6 horas, paralela al annulus y a una distancia mayor de 4 $\mathrm{mm}$ de éste, donde termina la porción cartilaginosa del conducto auditivo externo y comienza la ósea a modo de obtener un adecuado colgajo tímpano meatal. Se levanta un colgajo tímpano meatal cuidando no perforar el tímpano, se moviliza y preserva la cuerda del tímpano. Se observa la articulación incudoestapedial, las cruras del estribo, el tendón del músculo del estribo, la eminencia piramidal, el nicho de la ventana redonda, el promontorio y la porción timpánica del nervio facial. De ser necesario, se realiza fresado o curetaje de la pared póstero-superior del conducto auditivo externo con el objetivo de lograr mejor exposición. Se verifica fijación del estribo. Con respecto a los márgenes de seguridad, éstos son: el nervio facial por superior, la eminencia piramidal por posterior y la ventana redonda por inferior. Se realiza la medición de la prótesis desde la platina hasta la rama larga del yunque. Se realiza la platinostomía con fresa de 0,8 mm con el Skeeter drill ${ }^{\circledR}$, preservando la supraestructura. Luego se secciona el músculo del estribo lo más cerca de la pirámide, se fracturan ambas cruras del estribo hacia el promontorio y se remueve la supraestructura de estribo. Posteriormente se instala prótesis, se utilizaron dos modelos Schuknecht -Type Piston and Wire, Fluoroplastic/Stainless Steel Medtronic y Stapes -Prothese, Platin/PTFE SPIGGLE and THEIS y posterior ajuste de la prótesis al yunque con pinza de Mc Gee. Para finalizar, se reposiciona el colgajo tímpano meatal que se deja cubierto con gelfoam en sus bordes.

Se registraron las siguientes variables:

\section{Sexo}

Edad (en años)

Año de cirugía

Tiempo operatorio (en minutos)

Tipo de abordaje quirúrgico microscópico versus endoscópico

Lateralidad de oído afectado

Lateralidad de oído operado

Tipo de hipoacusia (sensorioneural, conductiva o mixta)

- Promedio tonal puro (PTP) pre y posquirúrgico (en frecuencia de 500, 1.000, 2.000, $4.000 \mathrm{~Hz}$ )

Diferencia $(G A P)$ óseo-aérea prey posquirúrgica $(d B)$

Cierre de GAP óseo-aéreo $(\mathrm{dB})$

- Tipo de prótesis

- Presencia y tipo de complicaciones quirúrgicas

Cirugías de revisión

- Hallazgos intraoperatorios (supraestructura de estribo intacta o no).

El análisis estadístico de los datos, fue realizado con el programa estadístico STATA 13. Se 
realizó un análisis descriptivo, detallando frecuencias absolutas, medidas de tendencia central y porcentajes. Se realizó comparación de medias utilizando el test exacto de Fisher para las variables categóricas y la prueba t de Student para las variables continuas, que en los casos de no presentar distribución normal, se normalizaron previo al análisis, o bien, se utilizó el test de Mann-Whitney. Se consideró un valor de $p<0.05$ estadísticamente significativo en los análisis realizados.

\section{RESULTADOS}

Se realizaron en total 52 cirugías, de 45 pacientes. Se excluyen de los resultados 3 casos donde no se instaló prótesis debido a que finalmente los pacientes no tenían el diagnóstico de otoesclerosis obteniendo un total de 49 cirugías, siendo el $75,51 \%$ de sexo femenino (37 pacientes) y $24,49 \%$ de sexo masculino (12 pacientes). El promedio de edad fue de 47,88 años (desviación estándar (DS) 10,03 ), con un rango de 24 a 69 años. De los casos incluidos el $79,19 \%$ presentaban una otoesclerosis bilateral, $12,5 \%$ otoesclerosis del oído derecho (OD) y $8,33 \%$ otoesclerosis del oído izquierdo (OI). Presentando una hipoacusia de conducción $33,33 \%$ y una hipoacusia mixta el $66,67 \%$ de los casos. Del total de las cirugías, el $89,8 \%$ fue por medio de un abordaje microscópico (44 cirugías) y 10,2\% por vía endoscópica (5 cirugías). El 68,22\% de las cirugías fue realizada en el OD y $37,78 \%$ en el Ol. El tiempo quirúrgico promedio fue de 127 minutos ( $D S=39,39$ ), con un rango de 25 a 225 minutos [25-225]. No se observaron diferencias significativas entre el tipo de abordaje y el tiempo quirúrgico (Mann-Whitney; $p=0,478$ ) (Tabla 1).

De las cirugías evaluadas, 5 fueron cirugías de revisión de estapedostomía antigua (10,2\%), de las cuales en 3 se encontraron bridas que fijaban la prótesis, 1 prótesis suelta que erosionó el yunque y 1 prótesis más larga de lo necesario (Tabla 2 ). Dentro de los hallazgos intraoperatorios de las cirugías que no fueron de revisión, 6,12\% presentó un nervio facial dehiscente y $2,04 \%$ presentó un nervio facial procidente (Tabla 3 ).

Estos resultados son más bajos en comparación con la literatura, y se podría explicar por ser diferentes cirujanos los cuales, no todos descri- bieron los hallazgos en los protocolos. Respecto al tipo de prótesis, se realizaron 30 cirugías con la prótesis Schuknecht -Type Piston and Wire, Medtronic y 19 cirugías con la prótesis Stapes-Prothese, Spiggle and Theis. Respecto a los resultados audiológicos, el PTP promedio preoperatorio fue de 64,71 (DS =18,61) decibeles (dB), con un gap promedio de 35,65 (DS =11,09, [15-79]) dB, siendo el PTP posoperatorio promedio de 36,57 (DS $=20,17) \mathrm{dB}$, con un gap posoperatorio promedio 11,34 (DS =19,19, [0-120], mediana =7). El cierre de gap promedio fue de $30,58 \mathrm{~dB}$ ( $D S=20,14$; mediana $=28,[0-120]$ ). Se presentó cofosis en 1 caso. Se encontraron diferencias significativas entre el PTP pre y posoperatorio $(p=0.000)$ y para el gap pre y posoperatorio $(p=0.000)$, evaluado por $t$-student (Figuras 1 y 2 ). Logrando un gap posoperatorio menos de $10 \mathrm{~dB}$ en el $78,57 \%$ de los casos, entre 11 y $20 \mathrm{~dB}$ en $11,9 \%$, entre 21 y $30 \mathrm{~dB}$ en $4,76 \%$, y mayor a $30 \mathrm{~dB}$ en $4,76 \%$, donde sólo 3 casos no logró cierre de gap. Respecto al cierre de gap en relación a la técnica de abordaje quirúrgico, no se observó diferencias significativas (MannWhitney; $p=0,189$ ) (Tabla 1 y Tabla 4).

\section{Tabla 1. Comparación de diferentes técnicas de abordaje quirúrgico}

\begin{tabular}{|lc|}
\hline $\begin{array}{l}\text { Diferencias entre técnica de abordaje } \\
\text { microscópica versus endoscópica }\end{array}$ & p-value \\
\hline Tiempo quirúrgico & 0,478 \\
Cierre de GAP & 0,189 \\
Complicaciones & 0,655 \\
\hline
\end{tabular}

Tabla 2. Hallazgos intraoperatorios

\begin{tabular}{|lc|}
\hline Hallazgo & Frecuencia \\
\hline Bridas & 3 \\
Prótesis suelta & 1 \\
Prótesis larga & 1 \\
\hline
\end{tabular}

Tabla 3. Hallazgos intraoperatorios

\begin{tabular}{|lc|}
\hline Hallazgo & Frecuencia \\
\hline Nervio facial dehiscente & 3 \\
Nervio facial procidente & 1 \\
\hline
\end{tabular}




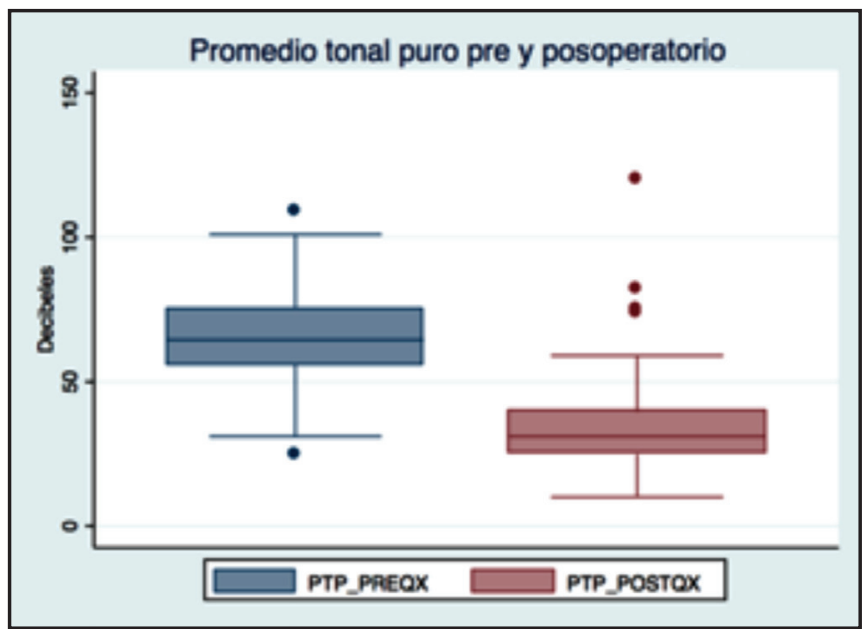

Figura 1. Cambio en el promedio tonal puro pre y posoperatorio.

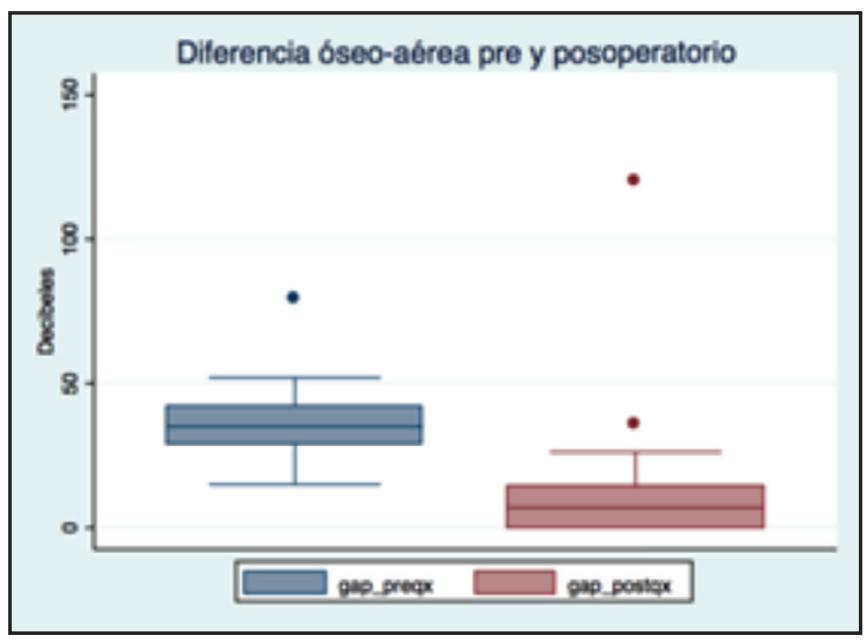

Figura 2. Cambio en la diferencia óseo-aérea entre el pre y posoperatorio.

Tabla 4. Resultados audiológicos

\begin{tabular}{|lcc|}
\hline Variable & $\begin{array}{c}\text { Preoperatorio }(\mathrm{dB}) \\
\text { (Promedio- DS) }\end{array}$ & $\begin{array}{c}\text { Posoperatorio }(\mathrm{dB}) \\
\text { (Promedio- DS) }\end{array}$ \\
\hline PTP & $64,72(18,61)$ & $36,57(20,17)$ \\
GAP & $35,65(11,09)$ & $11,34(19,18)$ \\
Cierre de GAP & $30,58(20,14)$ & \\
\hline
\end{tabular}

En cuanto a las complicaciones quirúrgicas, se observaron en $18,37 \%$, en 5 casos hubo sección de la cuerda del tímpano, 4 casos presentaron vértigo posoperatorio transitorio, en dos casos hubo platina flotante (colocándose injerto de grasa alrededor de la prótesis), en 2 casos hubo perforación del colgajo, en 1 caso hubo fractura de platina, en 1 caso hubo fístula perilinfática (que se corrigió inmediatamente en el intraoperatorio), en 1 caso presentó vértigo persistente (45 días posoperatorio) con signo de Tullio positivo, el cual fue necesario reintervenir y cambiar la prótesis por una de pistón más pequeño, y 1 caso presentó anacusia posoperatoria, el cual a pesar de realizar toda la técnica en la forma descrita y no presentar ninguna complicación intraoperatoria, evolucionó con anacusia, en este caso sospechamos que la platinostomía podría haber sido muy traumática para la cóclea. No hubo diferencias significativas 
entre la técnica de abordaje quirúrgico y la presencia de complicaciones (test exacto de Fisher; $p$ $=0,655)$. (Tablas 1 y 5 ).

\section{DISCUSIÓN}

El $87 \%$ de los pacientes intervenidos lograron éxito quirúrgico definido como cierre del gap óseo-aéreo $\leq 10 \mathrm{~dB}$ y/o mejoría en la conducción aérea en $20 \mathrm{~dB} 0$ menos. Estos resultados son comparables a las tasas de éxito reportadas tanto en la literatura nacional como internacional6-8. Con respecto a los casos donde no se instaló prótesis, en dos de ellos fue por malformaciones de cadena y uno por fístula perilinfática de alto flujo. Ambos casos son antiguos por lo que no se contaba con la disponibilidad de tomografía computarizada que existe actualmente en nuestro centro asistencial. Las dificultades quirúrgicas encontradas fueron similares a las publicadas en otras series y salvo en un caso, no impidieron la correcta implantación de prótesis. La tasa de complicaciones si bien es mayor que la publicada en series nacionales, el $67 \%$ de éstas fueron transitorias y los pacientes

\section{BIBLIOGRAFÍA}

1. Stott C, OJeda A, Muñoz D, Moyano L. Otoesclerosis. Aspectos histopatológicos y resultados auditivos de la estapedostomía. Rev Otorrinolaringol Cir Cabeza Cuello 2012; 72: 125-2.

2. Stott C, Arteaga P, Movano L. ¿Qué sabemos de otoesclerosis? Aspectos anatomopatológicos. Rev Otorrinolaringol Cir Cabeza Cuello 2005; 65: 179-6.

3. Casas J, Rodríguez D, Miranda G y cols. Otoesclerosis: Revisión de aspectos etiopatogénicos, clínicodemográficos e imagenológicos. Rev Chil Radiol 2016; 22: 3. doi: 10.1016/j.rchira.2016.08.002.

4. Délano P, Alvo A, Panussis F, Ojeda A, Stott C. Revisión de la experiencia clínica en el manejo quirúrgico de la otoesclerosis. Rev Hosp Clín Univ Chile 2011; 22: 305-9.
Tabla 5. Complicaciones quirúrgicas

\begin{tabular}{|lc|}
\hline Complicación & Frecuencia \\
\hline Sección cuerda del tímpano & 5 \\
Vértigo transitorio & 4 \\
Platina flotante & 2 \\
Fractura de platina & 1 \\
Fístula perilinfática & 1 \\
Vértigo persistente & 1 \\
Anacusia & 1 \\
\hline Total & 15 \\
\hline
\end{tabular}

fueron dados de alta asintomáticos. La paciente con vértigo persistente presentaba fenómeno de Tullio y fue sometida a cirugía de revisión y cambio de prótesis a una de menor tamaño, resolviendo así el vértigo.

\section{CONCLUSIÓN}

La estapedostomía es una intervención que si bien no está exenta de complicaciones, tanto en la literatura como en nuestra experiencia ha demostrado ser segura y tener buenos resultados auditivos en la mayoría de los pacientes.

5. Délano P, Alvo A, Ojeda A, Stott C. Resultados auditivos y hallazgos quirúrgicos en pacientes con cirugía bilateral por otoesclerosis. Rev Otorrinolaringol Cir Cabeza Cuello 2011; 71: 2016. doi:10.4067/S0718-48162011000300003.

6. Lanas A, Stott C, Olavarría C. Otoesclerosis: Resultados Auditivos de Estapedectomía y Estapedostomía. Rev Otorrinolaringol Cir Cabeza Cuello 2002; 62: 115-2.

7. Arias R, Silva M, Veloz M. Estapedostomía endoscópica: Experiencia en el Hospital del Salvador. Rev Otorrinolaringol Cir Cabeza Cuello 2015; 75: 122-8.

8. Kisilevsky VE, Dutt SN, Bailie NA, Halik JJ. Hearing results of 1145 stapedotomies evaluated with Amsterdam hearing evaluation plots. $J$ Laryngol Otol 2009.

\footnotetext{
Correspondencia: Leandro Rodríguez

Servicio de Otorrinolaringología, Hospital del Salvador,

Salvador 364, Providencia. Santiago, Chile

E mail: drleandrorodriguez@gmail.com
} 